\title{
Using Refined Theory to Studied Elastic Wave Scattering and Dynamic Stress Concentrations in Plates with Two Cutouts
}

\author{
Xujiao Yang, Zihe Li, Haoyu Liu* \\ Beihua University, Jilin, China \\ Email: *pipijiaojiao@qq.com
}

How to cite this paper: Yang, X.J., Li, Z.H. and Liu, H.Y. (2020) Using Refined Theory to Studied Elastic Wave Scattering and Dynamic Stress Concentrations in Plates with Two Cutouts. Journal of Applied Mathematics and Physics, 8, 2999-3018.

https://doi.org/10.4236/jamp.2020.812222

Received: November 10, 2020

Accepted: December 21, 2020

Published: December 24, 2020

Copyright ( 2020 by author(s) and Scientific Research Publishing Inc. This work is licensed under the Creative Commons Attribution International License (CC BY 4.0).

http://creativecommons.org/licenses/by/4.0/

\begin{abstract}
In this paper, based on complex variables and conformal mapping methods, using the refined dynamic equation of plates, elastic wave scattering and dynamic stress concentrations in plates with two cutouts were studied. Applying the orthogonal function expansion method, the problem to be solved can be reduced into the solution of a set of infinite algebraic equations. According to free boundary conditions, numerical results of dynamic moment concentration factors in thick plates with two circular cutouts analyze that: there will be more complex interaction changes between two-cutout situation than single cutout situation. In the case of low frequency or high frequency and thin plate, the hole-spacing in the absence of coupling interactions was larger or smaller. The numerical results and method can be used to analyze the dynamics and strength of plate-like structures.
\end{abstract}

\section{Keywords}

Refined Vibration Equation of Plate Bending, Complex Variable and Conformal Mapping Method, Two Holes, Elastic Wave Scattering and Dynamic Stress Concentrations

\section{Introduction}

Tablet is the typical components of aerospace, civil construction and other projects. To meet the operational needs, sometimes works must be open row of holes in the plate, because the interaction between holes, stress wave propagation in the board during the complex scattering phenomenon will occur, resulting in the dynamic stress concentration phenomenon near holes [1]. Because of the limitations of classical plate theory, when analyzing the stress concentration such 
as openings, elastic dynamics problem, it will produce some errors.

Reissner [2] first proposed the consideration of shear deformation plate theory equations of statics. Then, Mindlin [3] considered the transverse shear deformation and rotary inertia effects, and gave the plate bending dynamic equation for engineering practice. Pao, etc. [4] [5] first studied Mindlin plate with a single hole scattering of elastic waves and dynamic stress concentration problem, giving analytical solution. Liu's [6] complex variable method is following the former Soviet Union mechanics. Mushkhelishvili [7] proposed the complex function method is used to solve the two-dimensional static stress concentration problem; the proposed is an effective way for dynamic problem solving plane elasticity. The literature [8] [9] uses Liu's complex variable method, which is based on the Mindlin plate theory to solve the dynamic stress concentration of thick plate holes. Based on the analysis literature [10] of the plate bending vibration precision equation and the Mindlin plate equation, the results show that: The above based on Mindlin plate equation calculation results sometimes still produces large errors, and its research aspects are relatively simple, only considering the solution of a plane or a single cutout.

This paper based on the literature [11] gives precise equation of bending vibration plate, using Liu complex variable and conformal mapping method, studying the two openings on the flat elastic wave scattering and dynamic stress concentration and giving the general solution and the numerical results, and analyzes the wave number, thickness and pitch and other parameters on the dynamic moment of impact.

\section{Plate Bending Wave Equation and Its Solution}

According to the literature [11] flat dynamics theory, the plate bending wave control equation:

$$
\begin{gathered}
D \nabla^{2} \nabla^{2} W-(2-v) D T_{2}^{2} \nabla^{2} W+C T_{2}^{2} W+\left(\frac{7}{8}-v\right) D T_{2}^{4} W=0 \\
{\left[(1-2 \kappa) \nabla^{2}+T_{2}^{2}+\frac{8}{h^{2}}\right] F=-\left[(3-2 \kappa) \nabla^{2}+(1-2 \kappa) T_{2}^{2}-\frac{8}{h^{2}}\right] W} \\
\nabla^{2} f-\left(\frac{\pi^{2}}{h^{2}}+T_{2}^{2}\right) f=0
\end{gathered}
$$

In the formula, $C, D$ namely flat shear stiffness and flexural rigidity, $C=\frac{E h}{2(1+v)}, D=\frac{E h^{3}}{12\left(1-v^{2}\right)} ; E, v$ the elastic modulus and Poisson's ratio respectively; $\rho$ the density; $t$ the time; $\nabla^{2}$ the Laplace operator, $\nabla^{2}=\frac{\partial^{2}}{\partial x^{2}}+\frac{\partial^{2}}{\partial y^{2}}$; $T_{j}^{2}=\frac{1}{c_{j}^{2}} \frac{\partial^{2}}{\partial t^{2}},(j=1,2) ; c_{1}, c_{2}$ namely elastic compression and shear wave velocity, $c_{1}^{2}=\frac{\lambda+2 \mu}{\rho}, c_{2}^{2}=\frac{\mu}{\rho}$. 
Steady-state solution of the problem, let

$$
W=\tilde{W} \mathrm{e}^{-\mathrm{i} \omega t}, F=\tilde{F} \mathrm{e}^{-\mathrm{i} \omega t}, f=\tilde{f} \mathrm{e}^{-\mathrm{i} \omega t},
$$

Of which, $\omega$ is a flat circular frequency of the bending vibration.

In the following analysis omitted symbol on time factor and the unknown the potential function, by the Formula (1a) can be obtained flat lateral displacement function should satisfy the equation.

$$
\begin{gathered}
\nabla^{2} \nabla^{2} W+\frac{3-2 \kappa}{2(1-\kappa)} k_{2}^{2} \nabla^{2} W-\frac{3}{4(1-\kappa)} k_{2}^{4}\left(\frac{4}{k_{2}^{2} h^{2}}-\frac{\kappa}{6}-\frac{1}{3}\right) W=0, \\
\prod_{j=1}^{2}\left(\nabla^{2}+\alpha_{j}^{2}\right) W=0, \\
\nabla^{2} f-\alpha_{3}^{2} f=0 .
\end{gathered}
$$

Of which, $\kappa=\frac{1-2 v}{2(1-v)} ; \alpha_{j}(j=1,2)$ is the scattering wave number, Satisfies the equation of $\alpha^{4}-(2-v) k_{2}^{2} \alpha^{2}-6(1-v) k_{2}^{4}\left(\frac{1}{k_{2}^{2} h^{2}}-\frac{7-8 v}{48(1-v)}\right)=0, \quad \alpha_{3}^{2}=\frac{\pi^{2}}{h^{2}}-k_{2}^{2}$; $k_{j}^{2}=\omega^{2} / c_{j}^{2}(j=1,2)$.

At this point, the corresponding generalized displacement potential function $F(x, y)$ :

$$
F=F_{1}+F_{2}=\sum_{j=1}^{2}\left(\delta_{j}-1\right) W_{j}
$$

Of which, $\left(\nabla^{2}+\alpha_{j}^{2}\right) W_{j}=0 ; \delta_{j}(j=1,2)$ is the scale factor of displacement potential function, $\delta_{j}=\frac{16+2\left(\alpha_{j}^{2}-\kappa k_{2}^{2}\right) h^{2}}{8+\left[(3-2 \kappa) \alpha_{j}^{2}-k_{2}^{2}\right] h^{2}}$.

The general solution of wave Equation (1) can be described as:

$$
\begin{gathered}
W=\sum_{n=-\infty}^{\infty} \sum_{m=1}^{2} A_{n m} \mathrm{H}_{n}^{(1)}\left(\alpha_{m} r\right) \mathrm{e}^{\mathrm{i} n \beta}, \\
F=\sum_{n=-\infty}^{\infty} \sum_{m=1}^{2} A_{n m}\left(\delta_{m}-1\right) \mathrm{H}_{n}^{(1)}\left(\alpha_{m} r\right) \mathrm{e}^{\mathrm{i} n \beta}, \\
f=\sum_{n=-\infty}^{\infty} B_{n} \mathrm{~K}_{n}\left(\alpha_{3} r\right) \mathrm{e}^{\mathrm{i} n \beta} .
\end{gathered}
$$

Of which, $\mathrm{H}_{n}^{(1)}(\cdot)$ is the first kind Henkel function; $\mathrm{K}_{n}(\cdot)$ is Bessel function of imaginary argument [12]; $A_{n m}(m=1,2)$ and $B_{n}$ are a generalized displacement mode coefficient determined by the hole boundary conditions.

Flat structure generalized expression for the force:

$$
\begin{aligned}
M_{x}= & -D(1-v)\left[\sum_{j=1}^{2}\left(\delta_{j}-1\right) \frac{\partial^{2} W_{j}}{\partial x^{2}}+\frac{\partial^{2} f}{\partial x \partial y}\right] \\
& -\frac{1}{2} D v\left[\left(\nabla^{2}+k_{2}^{2}\right) W+\sum_{j=1}^{2}\left(\delta_{j}-1\right) \nabla^{2} W_{j}\right],
\end{aligned}
$$




$$
\begin{gathered}
M_{y}=-D(1-v)\left[\sum_{j=1}^{2}\left(\delta_{j}-1\right) \frac{\partial^{2} W_{j}}{\partial y^{2}}-\frac{\partial^{2} f}{\partial x \partial y}\right] \\
-\frac{1}{2} D v\left[\left(\nabla^{2}+k_{2}^{2}\right) W+\sum_{j=1}^{2}\left(\delta_{j}-1\right) \nabla^{2} W_{j}\right], \\
M_{x y}=M_{y x}=-D(1-v)\left[\sum_{j=1}^{2}\left(\delta_{j}-1\right) \frac{\partial^{2} W_{j}}{\partial x \partial y}-\frac{1}{2}\left(\frac{\partial^{2} f}{\partial x^{2}}-\frac{\partial^{2} f}{\partial y^{2}}\right)\right], \\
Q_{x}=-C\left[\sum_{j=1}^{2}\left(\delta_{j}-1\right) \frac{\partial W_{j}}{\partial x}+\frac{\partial f}{\partial y}\right]+C \frac{\partial W}{\partial x}+\frac{1}{4} D\left\{v\left(\nabla^{2}+k_{2}^{2}\right) \frac{\partial W}{\partial x}\right. \\
\left.+\left[(2-v) \nabla^{2}+(1-v) k_{2}^{2}\right] \sum_{j=1}^{2}\left(\delta_{j}-1\right) \frac{\partial W_{j}}{\partial x}+(1-v)\left(\nabla^{2}+k_{2}^{2}\right) \frac{\partial f}{\partial y}\right\}, \\
Q_{y}=-C\left[\sum_{j=1}^{2}\left(\delta_{j}-1\right) \frac{\partial W_{j}}{\partial y}-\frac{\partial f}{\partial x}\right]+C \frac{\partial W}{\partial y}+\frac{1}{4} D\left\{v\left(\nabla^{2}+k_{2}^{2}\right) \frac{\partial W}{\partial y}\right. \\
+
\end{gathered}
$$

Using a complex variable method, a complex variable function corresponds to a pair of real-valued functions with two real variables, the introduction of complex variables: $z=x+\mathrm{i} y ; \bar{z}=x-\mathrm{i} y$. By the Formula (6), by substitution, can be obtained after finishing:

$$
\begin{gathered}
M_{x}+M_{y}=-D \sum_{j=1}^{2}\left(\delta_{j}-1\right) \nabla^{2} W_{j}-D v \nabla^{2} W-D v k_{2}^{2} W \\
M_{y}-M_{x}+2 \mathrm{i} M_{x y}=4 D(1-v) \sum_{j=1}^{2}\left(\delta_{j}-1\right) \frac{\partial^{2}}{\partial z^{2}} W_{j}+4 D \mathrm{i}(1-v) \frac{\partial^{2}}{\partial z^{2}} f \\
Q_{x}-\mathrm{i} Q_{y}=-2 C \frac{\partial}{\partial z} \sum_{j=1}^{2}\left[\left(\delta_{j}-1\right) W_{j}-W+\mathrm{i} f\right]+\frac{1}{2} D \frac{\partial}{\partial z}\left\{v\left(\nabla^{2}+k_{2}^{2}\right) W\right. \\
\left.+\left[(2-v) \nabla^{2}+(1-v) k_{2}^{2}\right] \sum_{j=1}^{2}\left(\delta_{j}-1\right) W_{j}+\mathrm{i}(1-v)\left(\nabla^{2}+k_{2}^{2}\right) f\right\} .
\end{gathered}
$$

Among them, $\nabla^{2}=4 \frac{\partial^{2}}{\partial z \partial \bar{z}}$.

In the solution of plates flexural wave scattering and dynamic stress concentration problems in the hole of arbitrarily-shaped, the conformal mapping technique can be used, the Z-plane non-circular openings $L$ boundary Outland (within the domain) mapped into $\eta$ plane boundary $S$ outside the domain of the unit circle (within the domain). The mapping function is preferably the following form:

$$
z=\Omega(\eta)=c \eta+\psi(\eta)
$$

Of which, $\psi(\eta)$ is a holomorphic function.

In polar coordinates $(r, \beta)$, the Formula (7) can be written as

$$
M_{r}+M_{\beta}=-D \sum_{j=1}^{2}\left(\delta_{j}-1\right) \nabla^{2} W_{j}-D \nu \nabla^{2} W-D \nu k_{2}^{2} W,
$$




$$
\begin{gathered}
M_{\beta}-M_{r}+2 \mathrm{i} M_{r \beta}=\left\{4 D(1-v) \frac{\partial^{2}}{\partial z^{2}}\left[\sum_{j=1}^{2}\left(\delta_{j}-1\right) W_{j}+\mathrm{i} f\right]\right\} \exp (2 \mathrm{i} \beta), \\
Q_{r}-\mathrm{i} Q_{\beta}=\left\{-2 C \frac{\partial}{\partial z}\left[\sum_{j=1}^{2}\left(\delta_{j}-1\right) W_{j}-W+\mathrm{i} f\right]+\frac{1}{2} D \frac{\partial}{\partial z}\left\{v\left(\nabla^{2}+k_{2}^{2}\right) W\right.\right. \\
\left.+\left[(2-v) \nabla^{2}+(1-v) k_{2}^{2}\right] \sum_{j=1}^{2}\left(\delta_{j}-1\right) W_{j}+\mathrm{i}(1-v)\left(\nabla^{2}+k_{2}^{2}\right) f\right\} \exp (\mathrm{i} \beta) .
\end{gathered}
$$

Thus, in the $\eta=\rho \mathrm{e}^{\mathrm{i} \theta}$ plane, the Formula (9) can be rewritten as

$$
\begin{aligned}
M_{\rho}+M_{\theta}=- & \frac{4 D}{\left|\Omega^{\prime}(\eta)\right|^{2}} \sum_{j=1}^{2}\left(\delta_{j}-1\right) \frac{\partial^{2} W_{j}}{\partial \eta \partial \bar{\eta}}-\frac{4 D v}{\left|\Omega^{\prime}(\eta)\right|^{2}} \frac{\partial^{2} W}{\partial \eta \partial \bar{\eta}}-D v k_{2}^{2} W \\
M_{\theta}-M_{\rho}+2 \mathrm{i} M_{\rho \theta} & =\frac{4 D(1-v) \eta^{2}}{\rho^{2} \overline{\Omega^{\prime}(\eta)}} \frac{\partial}{\partial \eta}\left\{\frac{1}{\Omega^{\prime}(\eta)} \frac{\partial}{\partial \eta}\left[\sum_{j=1}^{2}\left(\delta_{j}-1\right) W_{j}+\mathrm{i} f\right]\right\}, \\
Q_{\rho}-\mathrm{i} Q_{\theta}= & \frac{-2 C \eta}{\rho\left|\Omega^{\prime}(\eta)\right|} \frac{\partial}{\partial \eta}\left[\sum_{j=1}^{2}\left(\delta_{j}-1\right) W_{j}-W+\mathrm{i} f\right] \\
& +\frac{2 D \eta}{\rho\left|\Omega^{\prime}(\eta)\right|} \frac{\partial}{\partial \eta}\left\{v\left(\frac{1}{\left|\Omega^{\prime}(\eta)\right|^{2}} \frac{\partial^{2} W}{\partial \eta \partial \bar{\eta}}+\frac{k_{2}^{2}}{4} W\right)\right. \\
& +\sum_{j=1}^{2}\left(\delta_{j}-1\right)\left[\frac{2-v}{\left|\Omega^{\prime}(\eta)\right|^{2}} \frac{\partial^{2} W_{j}}{\partial \eta \partial \bar{\eta}}+(1-v) \frac{k_{2}^{2}}{4} W_{j}\right] \\
& \left.+\mathrm{i}(1-v)\left(\frac{1}{\left|\Omega^{\prime}(\eta)\right|^{2}} \frac{\partial^{2} f}{\partial \eta \partial \bar{\eta}}+\frac{k_{2}^{2}}{4} f\right)\right\} .
\end{aligned}
$$

The Formula (10a) minus (10b), can be obtained:

$$
\begin{aligned}
M_{\rho}-\mathrm{i} M_{\rho \theta}= & -\frac{2 D}{\left|\Omega^{\prime}(\eta)\right|^{2}} \sum_{j=1}^{2}\left(\delta_{j}-1\right) \frac{\partial^{2} W_{j}}{\partial \eta \partial \bar{\eta}}-\frac{2 D v}{\left|\Omega^{\prime}(\eta)\right|^{2}} \frac{\partial^{2} W}{\partial \eta \partial \bar{\eta}}-\frac{D v}{2} k_{2}^{2} W \\
& -\frac{2 D(1-v) \eta^{2}}{\rho^{2} \overline{\Omega^{\prime}(\eta)}} \frac{\partial}{\partial \eta}\left\{\frac{1}{\Omega^{\prime}(\eta)} \frac{\partial}{\partial \eta}\left[\sum_{j=1}^{2}\left(\delta_{j}-1\right) W_{j}+\mathrm{i} f\right]\right\}, \\
M_{\rho}+\mathrm{i} M_{\rho \theta}= & -\frac{2 D}{\left|\Omega^{\prime}(\eta)\right|^{2}} \sum_{j=1}^{2}\left(\bar{\delta}_{j}-1\right) \frac{\partial^{2} W_{j}}{\partial \eta \partial \bar{\eta}}-\frac{2 D v}{\left|\Omega^{\prime}(\eta)\right|^{2}} \frac{\partial^{2} W}{\partial \eta \partial \bar{\eta}}-\frac{D v}{2} k_{2}^{2} W \\
& -\frac{2 D(1-v) \bar{\eta}^{2}}{\rho^{2} \Omega^{\prime}(\eta)} \frac{\partial}{\partial \bar{\eta}}\left\{\frac{1}{\overline{\Omega^{\prime}(\eta)}} \frac{\partial}{\partial \bar{\eta}}\left[\sum_{j=1}^{2}\left(\bar{\delta}_{j}-1\right) W_{j}-\mathrm{i} f\right]\right\} .
\end{aligned}
$$

By the Formula (10c) can be obtained:

$$
\begin{aligned}
& Q_{\rho}=\frac{-C}{\rho \mid \Omega^{\prime}(\eta)}\left\{\eta \frac{\partial}{\partial \eta}\left[\sum_{j=1}^{2}\left(\delta_{j}-1\right) W_{j}-W+\mathrm{i} f\right]+\bar{\eta} \frac{\partial}{\partial \bar{\eta}}\left[\sum_{j=1}^{2}\left(\bar{\delta}_{j}-1\right) W_{j}-W-\mathrm{i} f\right]\right\} \\
& +\frac{D \eta}{\rho\left|\Omega^{\prime}(\eta)\right|} \frac{\partial}{\partial \eta}\left\{v\left(\frac{1}{\left|\Omega^{\prime}(\eta)\right|^{2}} \frac{\partial^{2} W}{\partial \eta \partial \bar{\eta}}+\frac{k_{2}^{2}}{4} W\right)+\mathrm{i}(1-v)\left(\frac{1}{\left|\Omega^{\prime}(\eta)\right|^{2}} \frac{\partial^{2} f}{\partial \eta \partial \bar{\eta}}+\frac{k_{2}^{2}}{4} f\right)\right. \\
& \left.+\sum_{j=1}^{2}\left(\delta_{j}-1\right)\left[(2-v) \frac{1}{\left|\Omega^{\prime}(\eta)\right|^{2}} \frac{\partial^{2} W_{j}}{\partial \eta \partial \bar{\eta}}+(1-v) \frac{k_{2}^{2}}{4} W_{j}\right]\right\}
\end{aligned}
$$




$$
\begin{aligned}
& +\frac{D \bar{\eta}}{\rho\left|\Omega^{\prime}(\eta)\right|} \frac{\partial}{\partial \bar{\eta}}\left\{v\left(\frac{1}{\left|\Omega^{\prime}(\eta)\right|^{2}} \frac{\partial^{2} W}{\partial \eta \partial \bar{\eta}}+\frac{k_{2}^{2}}{4} W\right)-\mathrm{i}(1-v)\left(\frac{1}{\left|\Omega^{\prime}(\eta)\right|^{2}} \frac{\partial^{2} f}{\partial \eta \partial \bar{\eta}}+\frac{k_{2}^{2}}{4} f\right)\right. \\
& \left.+\sum_{j=1}^{2}\left(\bar{\delta}_{j}-1\right)\left[(2-v) \frac{1}{\left|\Omega^{\prime}(\eta)\right|^{2}} \frac{\partial^{2} W_{j}}{\partial \eta \partial \bar{\eta}}+(1-v) \frac{k_{2}^{2}}{4} W_{j}\right]\right\}
\end{aligned}
$$

\section{The Excitation of Incident Wave and Total Wave Field}

Studying of double hole case, without loss of generality, located at infinity incident a positive direction along the $\mathrm{x}$-axis plane wave propagation, omitting the time factor, and its expression is:

$$
\begin{gathered}
W^{(i)}=W_{0} \mathrm{e}^{\mathrm{i} \alpha_{1} x}=W_{0} \sum_{n=-\infty}^{\infty}(\mathrm{i})^{n} \mathrm{~J}_{n}\left(\alpha_{1} r\right) \mathrm{e}^{\mathrm{i} n \beta} \\
F^{(i)}=\left(\delta_{1}-1\right) W^{(i)} \\
f^{(i)}=0
\end{gathered}
$$

When solving the edge data of flat panel openings, in the flat panel, near each hole the total wave field should be superimposed together by incident field and scattered field generated by the various openings, namely the flat panel's displacement function and generalized displacement function can be expressed as

$$
\begin{gathered}
W=W^{(i)}+\sum_{m=1}^{2} W^{(s)}=W_{0} \mathrm{e}^{\mathrm{i} \alpha_{1} x}+\sum_{m=1}^{2} \sum_{n=-\infty}^{\infty} \sum_{j=1}^{2} A_{n j}^{m} \mathrm{H}_{n}^{(1)}\left(\alpha_{j}|\Omega(\eta)|\right)\left\{\frac{\Omega(\eta)}{\Omega(\eta) \mid}\right\}^{n} \\
F=F^{(i)}+\sum_{m=1}^{2} F^{(s)} \\
=\left(\delta_{1}-1\right) W_{0} \mathrm{e}^{\mathrm{i} \alpha_{1} x}+\sum_{m=1}^{2} \sum_{n=-\infty}^{\infty} \sum_{j=1}^{2} A_{n j}^{m}\left(\delta_{j}-1\right) \mathrm{H}_{n}^{(1)}\left(\alpha_{j}|\Omega(\eta)|\right)\left\{\frac{\Omega(\eta)}{|\Omega(\eta)|}\right\}^{n} \\
f=f^{(i)}+\sum_{m=1}^{2} f^{(s)}=\sum_{m=1}^{2} \sum_{n=-\infty}^{\infty} B_{n}^{m} \mathrm{~K}_{n}\left(\alpha_{3}|\Omega(\eta)|\right)\left\{\frac{\Omega(\eta)}{|\Omega(\eta)|}\right\}^{n}
\end{gathered}
$$

In the analysis and calculation, the generalized force component in each local polar coordinate system should be converted to polar coordinates.

\section{Boundary Conditions and Model Coefficients}

In the $\eta$ planar, let opening hole for free boundary conditions, each hole should meet the following three boundary conditions

$$
\left.M_{\rho}\right|_{\rho=1}=0,\left.M_{\rho \theta}\right|_{\rho=1}=0,\left.Q_{\rho}\right|_{\rho=1}=0 .
$$

From the Formulas (9) to (13) substituted into Formula (14), which satisfies the boundary conditions of the opening hole, the following expression can be obtained 


$$
\sum_{j=1}^{6} \sum_{n=-\infty}^{\infty} E_{n}^{i j} X_{n}^{j}=E_{i} \quad(i=1,2,3,4,5,6)
$$

Both ends of Formula (15) multiply by $\exp \left(-\mathrm{i} s \theta_{j}\right)$, and integrate into the interval $(-\pi, \pi)$, the infinite algebraic equations can be obtained as follows

$$
\sum_{n=-\infty}^{\infty} E_{n s} X_{n}=E_{s}
$$

Of which, $E_{n s}=\frac{1}{2 \pi} \int_{-\pi}^{\pi} E_{n} \exp \left(-\mathrm{i} s \theta_{j}\right) \mathrm{d} \theta_{j}, E_{s}=\frac{1}{2 \pi} \int_{-\pi}^{\pi} E_{i} \exp \left(-\mathrm{i} s \theta_{j}\right) \mathrm{d} \theta_{j}$

$$
E_{n}=\left[\begin{array}{llllll}
E_{11}^{n} & E_{12}^{n} & E_{13}^{n} & E_{14}^{n} & E_{15}^{n} & E_{16}^{n} \\
E_{21}^{n} & E_{22}^{n} & E_{23}^{n} & E_{24}^{n} & E_{25}^{n} & E_{26}^{n} \\
E_{31}^{n} & E_{32}^{n} & E_{33}^{n} & E_{34}^{n} & E_{35}^{n} & E_{36}^{n} \\
E_{41}^{n} & E_{42}^{n} & E_{43}^{n} & E_{44}^{n} & E_{45}^{n} & E_{46}^{n} \\
E_{51}^{n} & E_{52}^{n} & E_{53}^{n} & E_{54}^{n} & E_{55}^{n} & E_{56}^{n} \\
E_{61}^{n} & E_{62}^{n} & E_{63}^{n} & E_{64}^{n} & E_{65}^{n} & E_{66}^{n}
\end{array}\right], X_{n}^{j}=\left[\begin{array}{c}
A_{n 1}^{1} \\
A_{n 2}^{1} \\
B_{n}^{1} \\
A_{n 1}^{2} \\
A_{n 2}^{2} \\
B_{n}^{2}
\end{array}\right], E=\left[\begin{array}{c}
E_{1} \\
E_{2} \\
E_{3} \\
E_{4} \\
E_{5} \\
E_{6}
\end{array}\right]
$$

Of which,

$$
\begin{aligned}
& E_{1 j}^{n}=-\frac{(1-v) \alpha_{j}^{2} \eta_{1}^{2} \Omega^{\prime}\left(\eta_{1}\right)}{\overline{\Omega^{\prime}\left(\eta_{1}\right)}}\left(\delta_{j}-1\right) \mathrm{H}_{n-2}^{(1)}\left(\alpha_{j} r_{1}\right) \mathrm{e}^{\mathrm{i}(n-2) \theta_{1}} \\
& +2 \alpha_{j}^{2}\left[\operatorname{Re}\left(\delta_{j}\right)-1+v\left(1-\frac{k_{2}^{2}}{\alpha_{j}^{2}}\right)\right] \mathrm{H}_{n}^{(1)}\left(\alpha_{j} r_{1}\right) \mathrm{e}^{\mathrm{i} n \theta_{1}} \\
& -\frac{(1-v) \alpha_{j}^{2} \bar{\eta}_{1}^{2} \overline{\Omega^{\prime}\left(\eta_{1}\right)}}{\Omega^{\prime}\left(\eta_{1}\right)}\left(\bar{\delta}_{j}-1\right) \mathrm{H}_{n+2}^{(1)}\left(\alpha_{j} r_{1}\right) \mathrm{e}^{\mathrm{i}(n+2) \theta_{1}} \quad(j=1,2) \\
& E_{13}^{n}=\frac{-\mathrm{i}(1-v) \alpha_{3}^{2} \eta_{1}^{2} \Omega^{\prime}\left(\eta_{1}\right)}{\overline{\Omega^{\prime}\left(\eta_{1}\right)}} \mathrm{K}_{n-2}\left(\alpha_{3} r_{1}\right) \mathrm{e}^{\mathrm{i}(n-2) \theta_{1}} \\
& +\frac{\mathrm{i}(1-v) \alpha_{3}^{2} \bar{\eta}_{1}^{2} \overline{\Omega^{\prime}\left(\eta_{1}\right)}}{\Omega^{\prime}\left(\eta_{1}\right)} \mathrm{K}_{n+2}\left(\alpha_{3} r_{1}\right) \mathrm{e}^{\mathrm{i}(n+2) \theta_{1}} \\
& E_{1 j}^{n}=-\frac{(1-v) \alpha_{j-3}^{2} \eta_{1}^{2} \Omega^{\prime}\left(\eta_{1}\right)}{\overline{\Omega^{\prime}\left(\eta_{1}\right)}}\left(\delta_{j-3}-1\right) \mathrm{H}_{n-2}^{(1)}\left(\alpha_{j-3} r_{2}\right) \mathrm{e}^{\mathrm{i}(n-2) \theta_{2}} \\
& +2 \alpha_{j-3}^{2}\left[\operatorname{Re}\left(\delta_{j-3}\right)-1+v\left(1-\frac{k_{2}^{2}}{\alpha_{j-3}^{2}}\right)\right] \mathrm{H}_{n}^{(1)}\left(\alpha_{j-3} r_{2}\right) \mathrm{e}^{\mathrm{i} n \theta_{2}} \\
& -\frac{(1-v) \alpha_{j-3}^{2} \bar{\eta}_{1}^{2} \overline{\Omega^{\prime}\left(\eta_{1}\right)}}{\Omega^{\prime}\left(\eta_{1}\right)}\left(\bar{\delta}_{j-3}-1\right) \mathrm{H}_{n+2}^{(1)}\left(\alpha_{j-3} r_{2}\right) \mathrm{e}^{\mathrm{i}(n+2) \theta_{2}} \quad(j=4,5) \\
& E_{16}^{n}=\frac{-\mathrm{i}(1-v) \alpha_{3}^{2} \eta_{1}^{2} \Omega^{\prime}\left(\eta_{1}\right)}{\overline{\Omega^{\prime}\left(\eta_{1}\right)}} \mathrm{K}_{n-2}\left(\alpha_{3} r_{2}\right) \mathrm{e}^{\mathrm{i}(n-2) \theta_{2}} \\
& +\frac{\mathrm{i}(1-v) \alpha_{3}^{2} \bar{\eta}_{1}^{2} \overline{\Omega^{\prime}\left(\eta_{1}\right)}}{\Omega^{\prime}\left(\eta_{1}\right)} \mathrm{K}_{n+2}\left(\alpha_{3} r_{2}\right) \mathrm{e}^{\mathrm{i}(n+2) \theta_{2}} \\
& E_{2 j}^{n}=\frac{-\mathrm{i}(1-v) \alpha_{j}^{2} \eta_{1}^{2} \Omega^{\prime}\left(\eta_{1}\right)}{\overline{\Omega^{\prime}\left(\eta_{1}\right)}}\left(\delta_{j}-1\right) \mathrm{H}_{n-2}^{(1)}\left(\alpha_{j} r_{1}\right) \mathrm{e}^{\mathrm{i}(n-2) \theta_{1}}-2 \alpha_{j}^{2} \operatorname{Im}\left(\delta_{j}\right) \mathrm{H}_{n}^{(1)}\left(\alpha_{j} r_{1}\right) \mathrm{e}^{\mathrm{i} n \theta_{1}} \\
& +\frac{\mathrm{i}(1-v) \alpha_{j}^{2}{\overline{\eta_{1}}}^{2} \overline{\Omega^{\prime}\left(\eta_{1}\right)}}{\Omega^{\prime}\left(\eta_{1}\right)}\left(\bar{\delta}_{j}-1\right) \mathrm{H}_{n+2}^{(1)}\left(\alpha_{j} r_{1}\right) \mathrm{e}^{\mathrm{i}(n+2) \theta_{1}} \quad(j=1,2)
\end{aligned}
$$




$$
\begin{aligned}
& E_{23}^{n}=\frac{(1-v) \alpha_{3}^{2} \eta_{1}^{2} \Omega^{\prime}\left(\eta_{1}\right)}{\overline{\Omega^{\prime}\left(\eta_{1}\right)}} \mathrm{K}_{n-2}\left(\alpha_{3} r_{1}\right) \mathrm{e}^{\mathrm{i}(n-2) \theta_{1}} \\
& +\frac{(1-v) \alpha_{3}^{2} \bar{\eta}_{1}^{2} \overline{\Omega^{\prime}\left(\eta_{1}\right)}}{\Omega^{\prime}\left(\eta_{1}\right)} \mathrm{K}_{n+2}\left(\alpha_{3} r_{1}\right) \mathrm{e}^{\mathrm{i}(n+2) \theta_{1}} \\
& E_{2 j}^{n}=\frac{-\mathrm{i}(1-v) \alpha_{j-3}^{2} \eta_{1}^{2} \Omega^{\prime}\left(\eta_{1}\right)}{\overline{\Omega^{\prime}\left(\eta_{1}\right)}}\left(\delta_{j-3}-1\right) \mathrm{H}_{n-2}^{(1)}\left(\alpha_{j-3} r_{2}\right) \mathrm{e}^{\mathrm{i}(n-2) \theta_{2}} \\
& -2 \alpha_{j-3}^{2} \operatorname{Im}\left(\delta_{j-3}\right) \mathrm{H}_{n}^{(1)}\left(\alpha_{j-3} r_{2}\right) \mathrm{e}^{\mathrm{i} n \theta_{2}} \\
& +\frac{\mathrm{i}(1-v) \alpha_{j-3}^{2} \bar{\eta}_{1}^{2} \overline{\Omega^{\prime}\left(\eta_{1}\right)}}{\Omega^{\prime}\left(\eta_{1}\right)}\left(\bar{\delta}_{j-3}-1\right) \mathrm{H}_{n+2}^{(1)}\left(\alpha_{j-3} r_{2}\right) \mathrm{e}^{\mathrm{i}(n+2) \theta_{2}} \quad(j=4,5) \\
& E_{26}^{n}=\frac{(1-v) \alpha_{3}^{2} \eta_{1}^{2} \Omega^{\prime}\left(\eta_{1}\right)}{\overline{\Omega^{\prime}\left(\eta_{1}\right)}} \mathrm{K}_{n-2}\left(\alpha_{3} r_{2}\right) \mathrm{e}^{\mathrm{i}(n-2) \theta_{2}} \\
& +\frac{(1-v) \alpha_{3}^{2} \bar{\eta}_{1}^{2} \overline{\Omega^{\prime}\left(\eta_{1}\right)}}{\Omega^{\prime}\left(\eta_{1}\right)} \mathrm{K}_{n+2}\left(\alpha_{3} r_{2}\right) \mathrm{e}^{\mathrm{i}(n+2) \theta_{2}} \\
& E_{3 j}^{n}=\frac{-2 C \alpha_{j} \eta_{1} \Omega^{\prime}\left(\eta_{1}\right)}{D\left|\Omega^{\prime}\left(\eta_{1}\right)\right|}\left(\delta_{j}-2\right) \mathrm{H}_{n-1}^{(1)}\left(\alpha_{j} r_{1}\right) \mathrm{e}^{\mathrm{i}(n-1) \theta_{1}} \\
& +\frac{2 C \alpha_{j} \overline{\eta_{1}} \overline{\Omega^{\prime}\left(\eta_{1}\right)}}{D\left|\Omega^{\prime}\left(\eta_{1}\right)\right|}\left(\bar{\delta}_{j}-2\right) \mathrm{H}_{n+1}^{(1)}\left(\alpha_{j} r_{1}\right) \mathrm{e}^{\mathrm{i}(n+1) \theta_{1}} \\
& -\frac{\alpha_{j} \eta_{1} \Omega^{\prime}\left(\eta_{1}\right)}{2\left|\Omega^{\prime}\left(\eta_{1}\right)\right|}\left\{v\left(\alpha_{j}^{2}-k_{2}^{2}\right)+\left[(2-v) \alpha_{j}^{2}-(1-v) k_{2}^{2}\right]\right. \\
& \left.\times\left(\delta_{j}-1\right)\right\} \mathrm{H}_{n-1}^{(1)}\left(\alpha_{j} r_{1}\right) \mathrm{e}^{\mathrm{i}(n-1) \theta_{1}}+\frac{\alpha_{j} \overline{\eta_{1}} \overline{\Omega^{\prime}\left(\eta_{1}\right)}}{2\left|\Omega^{\prime}\left(\eta_{1}\right)\right|}\left\{v\left(\alpha_{j}^{2}-k_{2}^{2}\right)\right. \\
& \left.+\left[(2-v) \alpha_{j}^{2}-(1-v) k_{2}^{2}\right]\left(\bar{\delta}_{j}-1\right)\right\} \mathrm{H}_{n+1}^{(1)}\left(\alpha_{j} r_{1}\right) \mathrm{e}^{\mathrm{i}(n+1) \theta_{1}} \quad(j=1,2) \\
& E_{33}^{n}=\frac{2 \mathrm{i} C \eta_{1} \Omega^{\prime}\left(\eta_{1}\right)}{D\left|\Omega^{\prime}\left(\eta_{1}\right)\right|} \alpha_{3} \mathrm{~K}_{n-1}\left(\alpha_{3} r_{1}\right) \mathrm{e}^{\mathrm{i}(n-1) \theta_{1}} \\
& -\frac{2 \mathrm{i} C \overline{\eta_{1}} \overline{\Omega^{\prime}\left(\eta_{1}\right)}}{D\left|\Omega^{\prime}\left(\eta_{1}\right)\right|} \alpha_{3} \mathrm{~K}_{n+1}\left(\alpha_{3} r_{1}\right) \mathrm{e}^{\mathrm{i}(n+1) \theta_{1}} \\
& -\frac{\mathrm{i}(1-v) \eta_{1} \Omega^{\prime}\left(\eta_{1}\right)}{2\left|\Omega^{\prime}\left(\eta_{1}\right)\right|}\left(\alpha_{3}^{2}+k_{2}^{2}\right) \alpha_{3} \mathrm{~K}_{n-1}\left(\alpha_{3} r_{1}\right) \mathrm{e}^{\mathrm{i}(n-1) \theta_{1}} \\
& +\frac{\mathrm{i}(1-v) \overline{\eta_{1}} \overline{\Omega^{\prime}\left(\eta_{1}\right)}}{2\left|\Omega^{\prime}\left(\eta_{1}\right)\right|}\left(\alpha_{3}^{2}+k_{2}^{2}\right) \alpha_{3} \mathrm{~K}_{n+1}\left(\alpha_{3} r_{1}\right) \mathrm{e}^{\mathrm{i}(n+1) \theta_{1}} \\
& E_{3 j}^{n}=\frac{-2 C \alpha_{j-3} \eta_{1} \Omega^{\prime}\left(\eta_{1}\right)}{D\left|\Omega^{\prime}\left(\eta_{1}\right)\right|}\left(\delta_{j-3}-2\right) \mathrm{H}_{n-1}^{(1)}\left(\alpha_{j-3} r_{2}\right) \mathrm{e}^{\mathrm{i}(n-1) \theta_{2}} \\
& +\frac{2 C \alpha_{j-3} \overline{\eta_{1}} \overline{\Omega^{\prime}\left(\eta_{1}\right)}}{D\left|\Omega^{\prime}\left(\eta_{1}\right)\right|}\left(\bar{\delta}_{j-3}-2\right) \mathrm{H}_{n+1}^{(1)}\left(\alpha_{j-3} r_{2}\right) \mathrm{e}^{\mathrm{i}(n+1) \theta_{2}} \\
& -\frac{\alpha_{j-3} \eta_{1} \Omega^{\prime}\left(\eta_{1}\right)}{2\left|\Omega^{\prime}\left(\eta_{1}\right)\right|}\left\{v\left(\alpha_{j-3}^{2}-k_{2}^{2}\right)+\left[(2-v) \alpha_{j-3}^{2}-(1-v) k_{2}^{2}\right]\right.
\end{aligned}
$$




$$
\begin{aligned}
& \left.\times\left(\delta_{j-3}-1\right)\right\} \mathrm{H}_{n-1}^{(1)}\left(\alpha_{j-3} r_{2}\right) \mathrm{e}^{\mathrm{i}(n-1) \theta_{2}}+\frac{\alpha_{j-3} \overline{\eta_{1}} \overline{\Omega^{\prime}\left(\eta_{1}\right)}}{2\left|\Omega^{\prime}\left(\eta_{1}\right)\right|}\left\{v\left(\alpha_{j-3}^{2}-k_{2}^{2}\right)\right. \\
& \left.+\left[(2-v) \alpha_{j-3}^{2}-(1-v) k_{2}^{2}\right]\left(\bar{\delta}_{j-3}-1\right)\right\} \mathrm{H}_{n+1}^{(1)}\left(\alpha_{j-3} r_{2}\right) \mathrm{e}^{\mathrm{i}(n+1) \theta_{2}} \quad(j=4,5) \\
& E_{36}^{n}=\frac{2 \mathrm{i} C \eta_{1} \Omega^{\prime}\left(\eta_{1}\right)}{D\left|\Omega^{\prime}\left(\eta_{1}\right)\right|} \alpha_{3} \mathrm{~K}_{n-1}\left(\alpha_{3} r_{2}\right) \mathrm{e}^{\mathrm{i}(n-1) \theta_{2}} \\
& -\frac{2 \mathrm{i} C \overline{\eta_{1}} \overline{\Omega^{\prime}\left(\eta_{1}\right)}}{D\left|\Omega^{\prime}\left(\eta_{1}\right)\right|} \alpha_{3} \mathrm{~K}_{n+1}\left(\alpha_{3} r_{2}\right) \mathrm{e}^{\mathrm{i}(n+1) \theta_{2}} \\
& -\frac{\mathrm{i}(1-v) \eta_{1} \Omega^{\prime}\left(\eta_{1}\right)}{2\left|\Omega^{\prime}\left(\eta_{1}\right)\right|}\left(\alpha_{3}^{2}+k_{2}^{2}\right) \alpha_{3} \mathrm{~K}_{n-1}\left(\alpha_{3} r_{2}\right) \mathrm{e}^{\mathrm{i}(n-1) \theta_{2}} \\
& +\frac{\mathrm{i}(1-v) \overline{\eta_{1}} \overline{\Omega^{\prime}\left(\eta_{1}\right)}}{2\left|\Omega^{\prime}\left(\eta_{1}\right)\right|}\left(\alpha_{3}^{2}+k_{2}^{2}\right) \alpha_{3} \mathrm{~K}_{n+1}\left(\alpha_{3} r_{2}\right) \mathrm{e}^{\mathrm{i}(n+1) \theta_{2}} \\
& E_{4 j}^{n}=-\frac{(1-v) \alpha_{j}^{2} \eta_{2}^{2} \Omega^{\prime}\left(\eta_{2}\right)}{\overline{\Omega^{\prime}\left(\eta_{2}\right)}}\left(\delta_{j}-1\right) \mathrm{H}_{n-2}^{(1)}\left(\alpha_{j} r_{1}\right) \mathrm{e}^{\mathrm{i}(n-2) \theta_{1}} \\
& +2 \alpha_{j}^{2}\left[\operatorname{Re}\left(\delta_{j}\right)-1+v\left(1-\frac{k_{2}^{2}}{\alpha_{j}^{2}}\right)\right] \mathrm{H}_{n}^{(1)}\left(\alpha_{j} r_{1}\right) \mathrm{e}^{\mathrm{i} n \theta_{1}}
\end{aligned}
$$

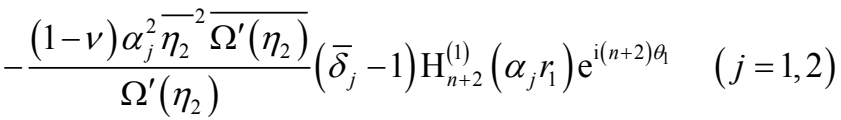

$$
\begin{aligned}
& E_{43}^{n}=\frac{-\mathrm{i}(1-v) \alpha_{3}^{2} \eta_{2}^{2} \Omega^{\prime}\left(\eta_{2}\right)}{\overline{\Omega^{\prime}\left(\eta_{2}\right)}} \mathrm{K}_{n-2}\left(\alpha_{3} r_{1}\right) \mathrm{e}^{\mathrm{i}(n-2) \theta_{1}} \\
& +\frac{\mathrm{i}(1-v) \alpha_{3}^{2}{\overline{\eta_{2}}}^{2} \overline{\Omega^{\prime}\left(\eta_{2}\right)}}{\Omega^{\prime}\left(\eta_{2}\right)} \mathrm{K}_{n+2}\left(\alpha_{3} r_{1}\right) \mathrm{e}^{\mathrm{i}(n+2) \theta_{1}} \\
& E_{4 j}^{n}=-\frac{(1-v) \alpha_{j-3}^{2} \eta_{2}^{2} \Omega^{\prime}\left(\eta_{2}\right)}{\overline{\Omega^{\prime}\left(\eta_{2}\right)}}\left(\delta_{j-3}-1\right) \mathrm{H}_{n-2}^{(1)}\left(\alpha_{j-3} r_{2}\right) \mathrm{e}^{\mathrm{i}(n-2) \theta_{2}} \\
& +2 \alpha_{j-3}^{2}\left[\operatorname{Re}\left(\delta_{j-3}\right)-1+v\left(1-\frac{k_{2}^{2}}{\alpha_{j-3}^{2}}\right)\right] \mathrm{H}_{n}^{(1)}\left(\alpha_{j-3} r_{2}\right) \mathrm{e}^{\mathrm{i} n \theta_{2}} \\
& -\frac{(1-v) \alpha_{j-3}^{2} \bar{\eta}_{2}^{2} \overline{\Omega^{\prime}\left(\eta_{2}\right)}}{\Omega^{\prime}\left(\eta_{2}\right)}\left(\bar{\delta}_{j-3}-1\right) \mathrm{H}_{n+2}^{(1)}\left(\alpha_{j-3} r_{2}\right) \mathrm{e}^{\mathrm{i}(n+2) \theta_{2}} \quad(j=4,5) \\
& E_{46}^{n}=\frac{-\mathrm{i}(1-v) \alpha_{3}^{2} \eta_{2}^{2} \Omega^{\prime}\left(\eta_{2}\right)}{\overline{\Omega^{\prime}\left(\eta_{2}\right)}} \mathrm{K}_{n-2}\left(\alpha_{3} r_{2}\right) \mathrm{e}^{\mathrm{i}(n-2) \theta_{2}} \\
& +\frac{\mathrm{i}(1-v) \alpha_{3}^{2} \bar{\eta}_{2}^{2} \overline{\Omega^{\prime}\left(\eta_{2}\right)}}{\Omega^{\prime}\left(\eta_{2}\right)} \mathrm{K}_{n+2}\left(\alpha_{3} r_{2}\right) \mathrm{e}^{\mathrm{i}(n+2) \theta_{2}} \\
& E_{5 j}^{n}=\frac{-\mathrm{i}(1-v) \alpha_{j}^{2} \eta_{2}^{2} \Omega^{\prime}\left(\eta_{2}\right)}{\overline{\Omega^{\prime}\left(\eta_{2}\right)}}\left(\delta_{j}-1\right) \mathrm{H}_{n-2}^{(1)}\left(\alpha_{j} r_{1}\right) \mathrm{e}^{\mathrm{i}(n-2) \theta_{1}} \\
& -2 \alpha_{j}^{2} \operatorname{Im}\left(\delta_{j}\right) \mathrm{H}_{n}^{(1)}\left(\alpha_{j} r_{1}\right) \mathrm{e}^{\mathrm{i} n \theta_{1}}
\end{aligned}
$$

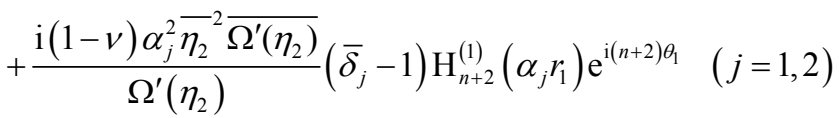




$$
\begin{aligned}
& E_{53}^{n}=\frac{(1-v) \alpha_{3}^{2} \eta_{2}^{2} \Omega^{\prime}\left(\eta_{2}\right)}{\overline{\Omega^{\prime}\left(\eta_{2}\right)}} \mathrm{K}_{n-2}\left(\alpha_{3} r_{1}\right) \mathrm{e}^{\mathrm{i}(n-2) \theta_{1}}
\end{aligned}
$$

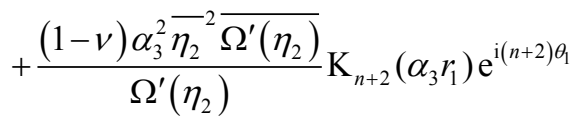

$$
\begin{aligned}
& E_{5 j}^{n}=\frac{-\mathrm{i}(1-v) \alpha_{j-3}^{2} \eta_{2}^{2} \Omega^{\prime}\left(\eta_{2}\right)}{\overline{\Omega^{\prime}\left(\eta_{2}\right)}}\left(\delta_{j-3}-1\right) \mathrm{H}_{n-2}^{(1)}\left(\alpha_{j-3} r_{2}\right) \mathrm{e}^{\mathrm{i}(n-2) \theta_{2}} \\
& -2 \alpha_{j-3}^{2} \operatorname{Im}\left(\delta_{j-3}\right) \mathrm{H}_{n}^{(1)}\left(\alpha_{j-3} r_{2}\right) \mathrm{e}^{\mathrm{i} n \theta_{2}} \\
& +\frac{\mathrm{i}(1-v) \alpha_{j-3}^{2} \bar{\eta}_{2}^{2} \overline{\Omega^{\prime}\left(\eta_{2}\right)}}{\Omega^{\prime}\left(\eta_{2}\right)}\left(\bar{\delta}_{j-3}-1\right) \mathrm{H}_{n+2}^{(1)}\left(\alpha_{j-3} r_{2}\right) \mathrm{e}^{\mathrm{i}(n+2) \theta_{2}} \quad(j=4,5) \\
& E_{53}^{n}=\frac{(1-v) \alpha_{3}^{2} \eta_{2}^{2} \Omega^{\prime}\left(\eta_{2}\right)}{\overline{\Omega^{\prime}\left(\eta_{2}\right)}} \mathrm{K}_{n-2}\left(\alpha_{3} r_{2}\right) \mathrm{e}^{\mathrm{i}(n-2) \theta_{2}} \\
& +\frac{(1-v) \alpha_{3}^{2}{\overline{\eta_{2}}}^{2} \overline{\Omega^{\prime}\left(\eta_{2}\right)}}{\Omega^{\prime}\left(\eta_{2}\right)} \mathrm{K}_{n+2}\left(\alpha_{3} r_{2}\right) \mathrm{e}^{\mathrm{i}(n+2) \theta_{2}} \\
& E_{6 j}^{n}=\frac{-2 C \alpha_{j} \eta_{2} \Omega^{\prime}\left(\eta_{2}\right)}{D\left|\Omega^{\prime}\left(\eta_{1}\right)\right|}\left(\delta_{j}-2\right) \mathrm{H}_{n-1}^{(1)}\left(\alpha_{j} r_{1}\right) \mathrm{e}^{\mathrm{i}(n-1) \theta_{1}} \\
& +\frac{2 C \alpha_{j} \overline{\eta_{2}} \overline{\Omega^{\prime}\left(\eta_{2}\right)}}{D\left|\Omega^{\prime}\left(\eta_{2}\right)\right|}\left(\bar{\delta}_{j}-2\right) \mathrm{H}_{n+1}^{(1)}\left(\alpha_{j} r_{1}\right) \mathrm{e}^{\mathrm{i}(n+1) \theta_{1}} \\
& -\frac{\alpha_{j} \eta_{2} \Omega^{\prime}\left(\eta_{2}\right)}{2\left|\Omega^{\prime}\left(\eta_{2}\right)\right|}\left\{v\left(\alpha_{j}^{2}-k_{2}^{2}\right)+\left[(2-v) \alpha_{j}^{2}-(1-v) k_{2}^{2}\right]\right. \\
& \left.\times\left(\delta_{j}-1\right)\right\} \mathrm{H}_{n-1}^{(1)}\left(\alpha_{j} r_{1}\right) \mathrm{e}^{\mathrm{i}(n-1) \theta_{1}}+\frac{\alpha_{j} \overline{\eta_{2}} \overline{\Omega^{\prime}\left(\eta_{2}\right)}}{2\left|\Omega^{\prime}\left(\eta_{2}\right)\right|}\left\{v\left(\alpha_{j}^{2}-k_{2}^{2}\right)\right. \\
& \left.+\left[(2-v) \alpha_{j}^{2}-(1-v) k_{2}^{2}\right]\left(\bar{\delta}_{j}-1\right)\right\} \mathrm{H}_{n+1}^{(1)}\left(\alpha_{j} r_{1}\right) \mathrm{e}^{\mathrm{i}(n+1) \theta_{1}} \quad(j=1,2) \\
& \left.E_{63}^{n}=\frac{2 \mathrm{i} C \eta_{2} \Omega^{\prime}\left(\eta_{2}\right)}{D\left|\Omega^{\prime}\left(\eta_{2}\right)\right|} \alpha_{3} \mathrm{~K}_{n-1}\left(\alpha_{3} r_{1}\right)\right) \mathrm{e}^{\mathrm{i}(n-1) \theta_{1}} \\
& -\frac{2 \mathrm{i} C \overline{\eta_{2}} \overline{\Omega^{\prime}\left(\eta_{2}\right)}}{D\left|\Omega^{\prime}\left(\eta_{2}\right)\right|} \alpha_{3} \mathrm{~K}_{n+1}\left(\alpha_{3} r_{1}\right) \mathrm{e}^{\mathrm{i}(n+1) \theta_{1}} \\
& -\frac{\mathrm{i}(1-v) \eta_{2} \Omega^{\prime}\left(\eta_{2}\right)}{2\left|\Omega^{\prime}\left(\eta_{2}\right)\right|}\left(\alpha_{3}^{2}+k_{2}^{2}\right) \alpha_{3} \mathrm{~K}_{n-1}\left(\alpha_{3} r_{1}\right) \mathrm{e}^{\mathrm{i}(n-1) \theta_{1}} \\
& +\frac{\mathrm{i}(1-v) \overline{\eta_{2}} \overline{\Omega^{\prime}\left(\eta_{2}\right)}}{2\left|\Omega^{\prime}\left(\eta_{2}\right)\right|}\left(\alpha_{3}^{2}+k_{2}^{2}\right) \alpha_{3} \mathrm{~K}_{n+1}\left(\alpha_{3} r_{1}\right) \mathrm{e}^{\mathrm{i}(n+1) \theta_{1}} \\
& E_{6 j}^{n}=\frac{-2 C \alpha_{j-3} \eta_{2} \Omega^{\prime}\left(\eta_{2}\right)}{D\left|\Omega^{\prime}\left(\eta_{2}\right)\right|}\left(\delta_{j-3}-2\right) \mathrm{H}_{n-1}^{(1)}\left(\alpha_{j-3} r_{2}\right) \mathrm{e}^{\mathrm{i}(n-1) \theta_{2}} \\
& +\frac{2 C \alpha_{j-3} \overline{\eta_{2}} \overline{\Omega^{\prime}\left(\eta_{2}\right)}}{D\left|\Omega^{\prime}\left(\eta_{2}\right)\right|}\left(\bar{\delta}_{j-3}-2\right) \mathrm{H}_{n+1}^{(1)}\left(\alpha_{j-3} r_{2}\right) \mathrm{e}^{\mathrm{i}(n+1) \theta_{2}} \\
& -\frac{\alpha_{j-3} \eta_{2} \Omega^{\prime}\left(\eta_{2}\right)}{2\left|\Omega^{\prime}\left(\eta_{2}\right)\right|}\left\{v\left(\alpha_{j-3}^{2}-k_{2}^{2}\right)+\left[(2-v) \alpha_{j-3}^{2}-(1-v) k_{2}^{2}\right]\right.
\end{aligned}
$$




$$
\begin{aligned}
& \left.\times\left(\delta_{j-3}-1\right)\right\} \mathrm{H}_{n-1}^{(1)}\left(\alpha_{j-3} r_{2}\right) \mathrm{e}^{\mathrm{i}(n-1) \theta_{2}}+\frac{\alpha_{j-3} \overline{\eta_{2}} \overline{\Omega^{\prime}\left(\eta_{2}\right)}}{2\left|\Omega^{\prime}\left(\eta_{2}\right)\right|}\left\{v\left(\alpha_{j-3}^{2}-k_{2}^{2}\right)\right. \\
& \left.+\left[(2-v) \alpha_{j-3}^{2}-(1-v) k_{2}^{2}\right]\left(\bar{\delta}_{j-3}-1\right)\right\} \mathrm{H}_{n+1}^{(1)}\left(\alpha_{j-3} r_{2}\right) \mathrm{e}^{\mathrm{i}(n+1) \theta_{2}} \quad(j=4,5) \\
& E_{66}^{n}=\frac{2 \mathrm{i} C \eta_{2} \Omega^{\prime}\left(\eta_{2}\right)}{D\left|\Omega^{\prime}\left(\eta_{2}\right)\right|} \alpha_{3} \mathrm{~K}_{n-1}\left(\alpha_{3} r_{2}\right) \mathrm{e}^{\mathrm{i}(n-1) \theta_{2}} \\
& -\frac{2 \mathrm{i} C \overline{\eta_{2}} \overline{\Omega^{\prime}\left(\eta_{2}\right)}}{D\left|\Omega^{\prime}\left(\eta_{2}\right)\right|} \alpha_{3} \mathrm{~K}_{n+1}\left(\alpha_{3} r_{2}\right) \mathrm{e}^{\mathrm{i}(n+1) \theta_{2}} \\
& -\frac{\mathrm{i}(1-v) \eta_{2} \Omega^{\prime}\left(\eta_{2}\right)}{2\left|\Omega^{\prime}\left(\eta_{2}\right)\right|}\left(\alpha_{3}^{2}+k_{2}^{2}\right) \alpha_{3} \mathrm{~K}_{n-1}\left(\alpha_{3} r_{2}\right) \mathrm{e}^{\mathrm{i}(n-1) \theta_{2}} \\
& +\frac{\mathrm{i}(1-v) \overline{\eta_{2}} \overline{\Omega^{\prime}\left(\eta_{2}\right)}}{2\left|\Omega^{\prime}\left(\eta_{2}\right)\right|}\left(\alpha_{3}^{2}+k_{2}^{2}\right) \alpha_{3} \mathrm{~K}_{n+1}\left(\alpha_{3} r_{2}\right) \mathrm{e}^{\mathrm{i}(n+1) \theta_{2}} \\
& E_{1}=-2 \alpha_{1}^{2}\left[\operatorname{Re}\left(\delta_{1}\right)-1+v\left(1-\frac{k_{2}^{2}}{\alpha_{1}^{2}}\right)\right] W_{0} \mathrm{e}^{\mathrm{i} \alpha_{1} \operatorname{Re}\left[\Omega\left(\eta_{1}\right)\right]} \\
& -2(1-v) \alpha_{1}^{2} \operatorname{Re}\left[\frac{\eta_{1}^{2} \Omega^{\prime}\left(\eta_{1}\right)}{\overline{\Omega^{\prime}\left(\eta_{1}\right)}}\left(\delta_{1}-1\right)\right] W_{0} \mathrm{e}^{\mathrm{i} \alpha_{1} \operatorname{Re}\left[\Omega\left(\eta_{1}\right)\right]} \\
& E_{2}=2 \alpha_{1}^{2} \operatorname{Im}\left(\delta_{1}\right) W_{0} \mathrm{e}^{\mathrm{i} \alpha_{1} \operatorname{Re}\left[\Omega\left(\eta_{1}\right)\right]} \\
& +2(1-v) \alpha_{1}^{2} \operatorname{Im}\left[\frac{\eta_{1}^{2} \Omega^{\prime}\left(\eta_{1}\right)}{\overline{\Omega^{\prime}\left(\eta_{1}\right)}}\left(\delta_{1}-1\right)\right] W_{0} \mathrm{e}^{\mathrm{i} \alpha_{1} \operatorname{Re}\left[\Omega\left(\eta_{1}\right)\right]} \\
& E_{3}=\frac{4 C}{D} \mathrm{i} \alpha_{1} \operatorname{Re}\left[\frac{\eta_{1} \Omega^{\prime}\left(\eta_{1}\right)}{\left|\Omega^{\prime}\left(\eta_{1}\right)\right|}\left(\delta_{1}-2\right)\right] W_{0} \mathrm{e}^{\mathrm{i} \alpha_{1} \operatorname{Re}\left[\Omega\left(\eta_{1}\right)\right]} \\
& +\mathrm{i} v\left(\alpha_{1}^{2}-k_{2}^{2}\right) \alpha_{1} \operatorname{Re}\left[\frac{\eta_{1} \Omega^{\prime}\left(\eta_{1}\right)}{\left|\Omega^{\prime}\left(\eta_{1}\right)\right|}\right] W_{0} \mathrm{e}^{\mathrm{i} \alpha_{1} \operatorname{Re}\left[\Omega\left(\eta_{1}\right)\right]} \\
& +\mathrm{i}\left[(2-v) \alpha_{1}^{2}-(1-v) k_{2}^{2}\right] \alpha_{1} \operatorname{Re}\left[\frac{\eta_{1} \Omega^{\prime}\left(\eta_{1}\right)}{\left|\Omega^{\prime}\left(\eta_{1}\right)\right|}\left(\delta_{1}-1\right)\right] W_{0} \mathrm{e}^{\mathrm{i} \alpha_{1} \operatorname{Re}\left[\Omega\left(\eta_{1}\right)\right]} \\
& E_{4}=-2 \alpha_{1}^{2}\left[\operatorname{Re}\left(\delta_{1}\right)-1+v\left(1-\frac{k_{2}^{2}}{\alpha_{1}^{2}}\right)\right] W_{0} \mathrm{e}^{\mathrm{i} \alpha_{1} \operatorname{Re}\left[\Omega\left(\eta_{2}\right)\right]} \\
& -2(1-v) \alpha_{1}^{2} \operatorname{Re}\left[\frac{\eta^{2} \Omega^{\prime}\left(\eta_{2}\right)}{\overline{\Omega^{\prime}\left(\eta_{2}\right)}}\left(\delta_{1}-1\right)\right] W_{0} \mathrm{e}^{\mathrm{i} \alpha_{1} \operatorname{Re}\left[\Omega\left(\eta_{2}\right)\right]} \\
& E_{5}=2 \alpha_{1}^{2} \operatorname{Im}\left(\delta_{1}\right) W_{0} \mathrm{e}^{\mathrm{i} \alpha_{1} \operatorname{Re}\left[\Omega\left(\eta_{2}\right)\right]} \\
& +2(1-v) \alpha_{1}^{2} \operatorname{Im}\left[\frac{\eta^{2} \Omega^{\prime}\left(\eta_{2}\right)}{\overline{\Omega^{\prime}\left(\eta_{2}\right)}}\left(\delta_{1}-1\right)\right] W_{0} \mathrm{e}^{\mathrm{i} \alpha_{1} \operatorname{Re}\left[\Omega\left(\eta_{2}\right)\right]} \\
& E_{6}=\frac{4 C}{D} \mathrm{i} \alpha_{1} \operatorname{Re}\left[\frac{\eta_{2} \Omega^{\prime}\left(\eta_{2}\right)}{\left|\Omega^{\prime}\left(\eta_{2}\right)\right|}\left(\delta_{1}-2\right)\right] W_{0} \mathrm{e}^{\mathrm{i} \alpha_{1} \operatorname{Re}\left[\Omega\left(\eta_{2}\right)\right]} \\
& +\mathrm{i} v\left(\alpha_{1}^{2}-k_{2}^{2}\right) \alpha_{1} \operatorname{Re}\left[\frac{\eta_{2} \Omega^{\prime}\left(\eta_{2}\right)}{\left|\Omega^{\prime}\left(\eta_{2}\right)\right|}\right] W_{0} \mathrm{e}^{\mathrm{i} \alpha_{1} \operatorname{Re}\left[\Omega\left(\eta_{2}\right)\right]} \\
& +\mathrm{i}\left[(2-v) \alpha_{1}^{2}-(1-v) k_{2}^{2}\right] \alpha_{1} \operatorname{Re}\left[\frac{\eta_{2} \Omega^{\prime}\left(\eta_{2}\right)}{\left|\Omega^{\prime}\left(\eta_{2}\right)\right|}\left(\delta_{1}-1\right)\right] W_{0} \mathrm{e}^{\mathrm{i} \alpha_{1} \operatorname{Re}\left[\Omega\left(\eta_{2}\right)\right]}
\end{aligned}
$$


Of which,

$$
\begin{gathered}
\frac{C}{D}=\frac{6(1-v)}{h^{2}} \\
\eta_{1}=\exp \left(\mathrm{i} a \theta_{1}\right), \quad r_{2}=\sqrt{a^{2}+d^{2}+2 a d \sin \theta_{1}}, \quad \theta_{2}=\arccos \frac{a \cos \theta_{1}}{r_{2}} \\
\eta_{2}=\exp \left(\mathrm{i} a \theta_{2}\right), \quad r_{1}=\sqrt{a^{2}+d^{2}-2 a d \sin \theta_{2}}, \quad \theta_{1}=-\arccos \frac{a \cos \theta_{2}}{r_{1}}
\end{gathered}
$$

By the definition of opening hole of dynamic stress concentration: dynamic stress concentration factor is the ratio of the hoop moving bending moment on the periphery of opening hole and the wave amplitude bending moment of the incident direction of the incident, that

$$
M_{\theta}^{*}=M_{\theta} / M_{0}
$$

Of which,

$$
\begin{gathered}
M_{\theta}=M_{\theta}^{(i)}+M_{\theta}^{(s)} \\
M_{\theta}^{(i)}=D \alpha_{1}^{2}\left[\delta_{1}-\frac{1}{2} \operatorname{Re}\left(\delta_{1}\right)-\frac{1}{2}(1-v)\right] W_{0} \mathrm{e}^{\mathrm{i} \alpha_{1} \mathrm{Re}[\Omega(\eta)]} \\
-\frac{1}{2} D v k_{2}^{2} W_{0} \mathrm{e}^{\mathrm{i} \alpha_{1} \mathrm{Re}[\Omega(\eta)]} \\
-D \frac{1-v}{2} \alpha_{1}^{2} \operatorname{Re}\left[\frac{\eta^{2} \Omega^{\prime}(\eta)}{\overline{\Omega^{\prime}(\eta)}}\left(\delta_{1}-1\right)\right] W_{0} \mathrm{e}^{\mathrm{i} \alpha_{1} \mathrm{Re}[\Omega(\eta)]} \\
M_{\theta}^{(s)}=D \sum_{j=1}^{2}\left[\delta_{j}-\frac{1}{2} \operatorname{Re}\left(\delta_{j}\right)-\frac{1}{2}+\frac{1}{2} v\left(1-\frac{k_{2}^{2}}{\alpha_{j}^{2}}\right)\right] \alpha_{j}^{2} \\
\times \sum_{n=-\infty}^{\infty} A_{n j} \mathrm{H}_{n}^{(1)}\left(\alpha_{j}|\Omega(\eta)|\right)\left\{\frac{\Omega(\eta)}{|\Omega(\eta)|}\right\}^{n} \\
+\frac{D(1-v) \eta^{2} \Omega^{\prime}(\eta)}{4 \overline{\Omega^{\prime}(\eta)}} \sum_{j=1}^{2}\left(\delta_{j}-1\right) \alpha_{j}^{2} \sum_{n=-\infty}^{\infty} A_{n j} \mathrm{H}_{n-2}^{(1)}\left(\alpha_{j}|\Omega(\eta)|\right)\left\{\frac{\Omega(\eta)}{|\Omega(\eta)|}\right\}^{n-2} \\
+\frac{D(1-v) \bar{\eta}^{2} \overline{\Omega^{\prime}(\eta)}}{4 \Omega^{\prime}(\eta)} \sum_{j=1}^{2}\left(\bar{\delta}_{j}-1\right) \alpha_{j}^{2} \sum_{n=-\infty}^{\infty} A_{n j} \mathrm{H}_{n+2}^{(1)}\left(\alpha_{j}|\Omega(\eta)|\right)\left\{\frac{\Omega(\eta)}{|\Omega(\eta)|}\right\}^{n+2} \\
+\frac{\mathrm{i} D(1-v) \eta^{2} \Omega^{\prime}(\eta)}{4 \overline{\Omega^{\prime}(\eta)}} \alpha_{3}^{2} \sum_{n=-\infty}^{\infty} B_{n} \mathrm{~K}_{n-2}\left(\alpha_{3}|\Omega(\eta)|\right)\left\{\frac{\Omega(\eta)}{|\Omega(\eta)|}\right\}^{n-2} D v\left[\left(\alpha_{1}^{2}-k_{2}^{2}\right)+(2-v)\left(\delta_{1}-1\right) \alpha_{1}^{2}\right] W_{0} \\
-\frac{\mathrm{i} D(1-v) \bar{\eta}^{2} \overline{\Omega^{\prime}(\eta)}}{4 \Omega^{\prime}(\eta)} \alpha_{3}^{2} \sum_{n=-\infty}^{\infty} B_{n} \mathrm{~K}_{n+2}\left(\alpha_{3}|\Omega(\eta)|\right)\left\{\frac{\Omega(\eta)}{|\Omega(\eta)|}\right\}^{n+2}
\end{gathered}
$$

Thus, for a flat with double holes, openings around the $m$-th dynamic stress concentration factor can be expressed as 


$$
\begin{aligned}
& M_{\theta}^{*}=D \alpha_{1}^{2}\left[\delta_{1}-\frac{1}{2} \operatorname{Re}\left(\delta_{1}\right)-\frac{1}{2}(1-v)\right] W_{0} \mathrm{e}^{\mathrm{i} \alpha_{1} \operatorname{Re}\left[\Omega\left(\eta_{m}\right)\right]}-\frac{1}{2} D v k_{2}^{2} W_{0} \mathrm{e}^{\mathrm{i} \alpha_{1} \operatorname{Re}\left[\Omega\left(\eta_{m}\right)\right]} \\
& -D \frac{1-v}{2} \alpha_{1}^{2} \operatorname{Re}\left[\frac{\eta_{m}^{2} \Omega^{\prime}\left(\eta_{m}\right)}{\overline{\Omega^{\prime}\left(\eta_{m}\right)}}\left(\delta_{1}-1\right)\right] W_{0} \mathrm{e}^{\mathrm{i} \alpha_{1} \operatorname{Re}\left[\Omega\left(\eta_{m}\right)\right]} \\
& +D \sum_{j=1}^{2}\left[\delta_{j}-\frac{1}{2} \operatorname{Re}\left(\delta_{j}\right)-\frac{1}{2}+\frac{1}{2} v\left(1-\frac{k_{2}^{2}}{\alpha_{j}^{2}}\right)\right] \alpha_{j}^{2} \sum_{n=-\infty}^{\infty} A_{n j}^{m} \mathrm{H}_{n}^{(1)}\left(\alpha_{j}\left|\Omega\left(\eta_{m}\right)\right|\right)\left\{\frac{\Omega\left(\eta_{m}\right)}{\left|\Omega\left(\eta_{m}\right)\right|}\right\}^{n} \\
& +D \frac{(1-v) \eta_{m}^{2} \Omega^{\prime}\left(\eta_{m}\right)}{4 \overline{\Omega^{\prime}\left(\eta_{m}\right)}} \sum_{j=1}^{2}\left(\delta_{j}-1\right) \alpha_{j}^{2} \sum_{n=-\infty}^{\infty} A_{n j}^{m} \mathrm{H}_{n-2}^{(1)}\left(\alpha_{j}\left|\Omega\left(\eta_{m}\right)\right|\right)\left\{\frac{\Omega\left(\eta_{m}\right)}{\Omega\left(\eta_{m}\right)}\right\}^{n-2} \\
& +D \frac{(1-v){\overline{\eta_{m}}}^{2} \overline{\Omega^{\prime}\left(\eta_{m}\right)}}{4 \Omega^{\prime}\left(\eta_{m}\right)} \sum_{j=1}^{2}\left(\bar{\delta}_{j}-1\right) \alpha_{j}^{2} \sum_{n=-\infty}^{\infty} A_{n j}^{m} \mathrm{H}_{n+2}^{(1)}\left(\alpha_{j}\left|\Omega\left(\eta_{m}\right)\right|\right)\left\{\frac{\Omega\left(\eta_{m}\right)}{\left|\Omega\left(\eta_{m}\right)\right|}\right\}^{n+2} \\
& +D \frac{\mathrm{i}(1-v) \eta_{m}^{2} \Omega^{\prime}(\eta)}{4 \overline{\Omega^{\prime}(\eta)}} \alpha_{3}^{2} \sum_{n=-\infty}^{\infty} B_{n}^{m} \mathrm{~K}_{n-2}\left(\alpha_{3}\left|\Omega\left(\eta_{m}\right)\right|\right)\left\{\frac{\Omega\left(\eta_{m}\right)}{\left|\Omega\left(\eta_{m}\right)\right|}\right\}^{n-2} \\
& -D \frac{\mathrm{i}(1-v){\overline{\eta_{m}}}^{2} \overline{\Omega^{\prime}\left(\eta_{m}\right)}}{4 \Omega^{\prime}\left(\eta_{m}\right)} \alpha_{3}^{2} \sum_{n=-\infty}^{\infty} B_{n}^{m} \mathrm{~K}_{n+2}\left(\alpha_{3}\left|\Omega\left(\eta_{m}\right)\right|\right)\left\{\frac{\Omega\left(\eta_{m}\right)}{\left|\Omega\left(\eta_{m}\right)\right|}\right\}^{n+2}
\end{aligned}
$$

Formula (19) is a general expression of $m$-th dynamic stress concentration factor for a flat with double holes.

\section{Numerical Results}

The above analysis process can be used to calculate the thick plate with two round hole for its hole dynamic stress concentration factor. According to the theory of plate exact formulas [6], the computational procedure for the flat plate with two round holes for its dynamic stress concentration factor can be compiled. According to the actual situation and empirical value, it is determined as, Poisson ratio $v=0.3$, dimensionless parameter's pore size and plate thickness ratio $a / h=0.1 \sim 2.0$, the wave number $k a=0.1 \sim 3.0$.

The distribution of dynamic moment's concentration coefficient shown in Figures 1-17, the upper hole is calculated between the hole on the upper and lower deployment hole in the figure, connecting the center of two holes perpendicular to the $x$-axis, and the center of the two holes' distance is $L$. The upper half of Figures 1-16 is the regular pattern of a single hole when $t=0$ the concentration factor of dynamic moment changed with the circumferential angle $(0 \sim \pi)$, and the lower half of Figures $1-16$ is the regular pattern of two round holes when $t=0$ the concentration factor of dynamic moment changed with the circumferential angle $(-\pi \sim 0)$. Figure 17 shows the double-hole between the dynamic moment concentration factor with dimensionless hole spacing changes in the law. Figure 17 is the regular pattern of the dynamic moment concentration factor between the double-hole changed with dimensionless variation of hole spacing $L / a$. 


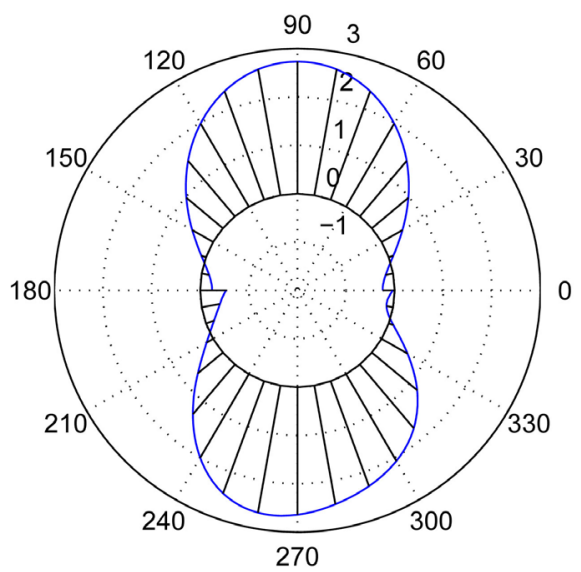

$$
(k a=0.1, a / h=0.1, L / a=2.1)
$$

Figure 1. Dynamic moment factor 1.

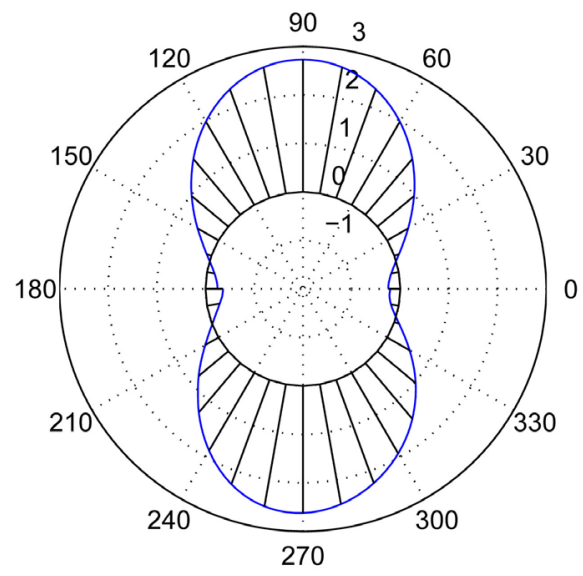

$$
(k a=0.1, a / h=0.1, L / a=2.5)
$$

Figure 2. Dynamic moment factor 2.

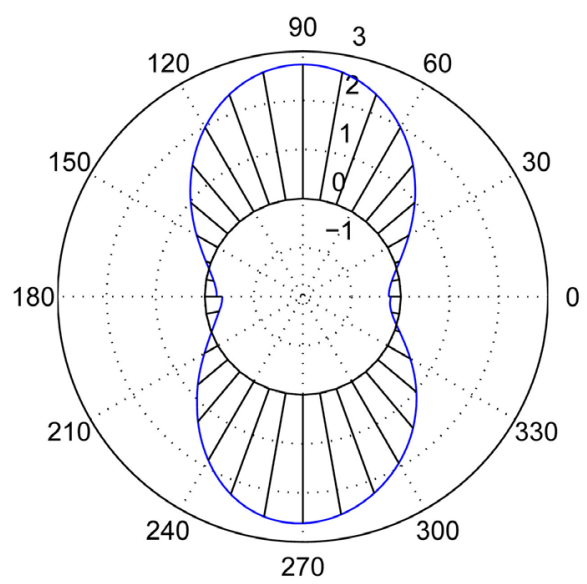

$$
(k a=0.1, a / h=0.1, L / a=4.0)
$$

Figure 3. Dynamic moment factor 3. 


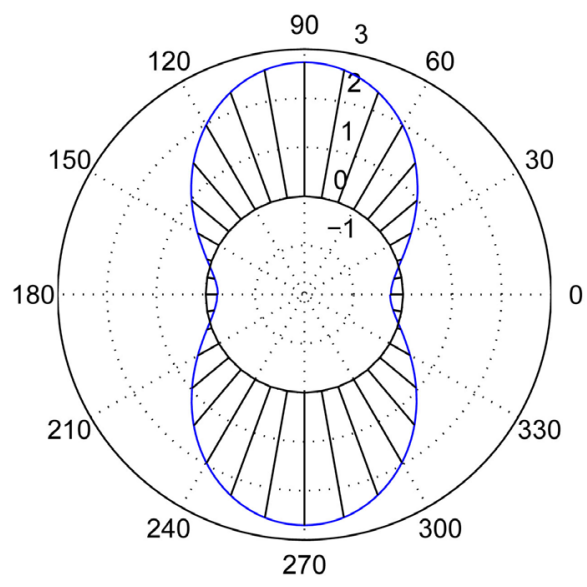

$$
(k a=0.1, a / h=0.1, L / a=6.0)
$$

Figure 4. Dynamic moment factor 4.

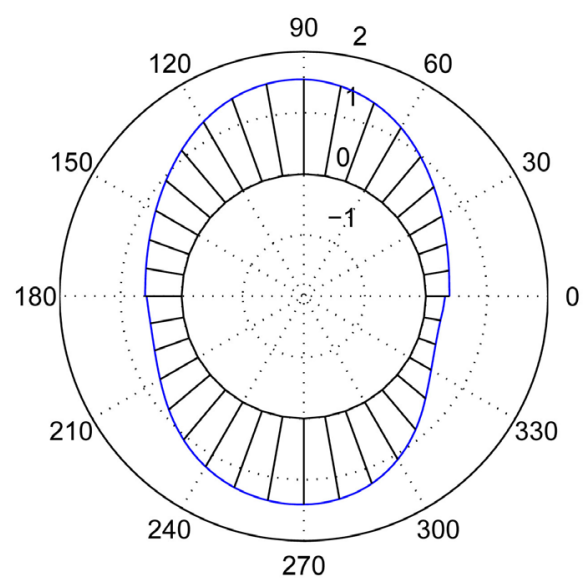

$$
(k a=0.5, a / h=0.5, L / a=2.1)
$$

Figure 5. Dynamic moment factor 5.

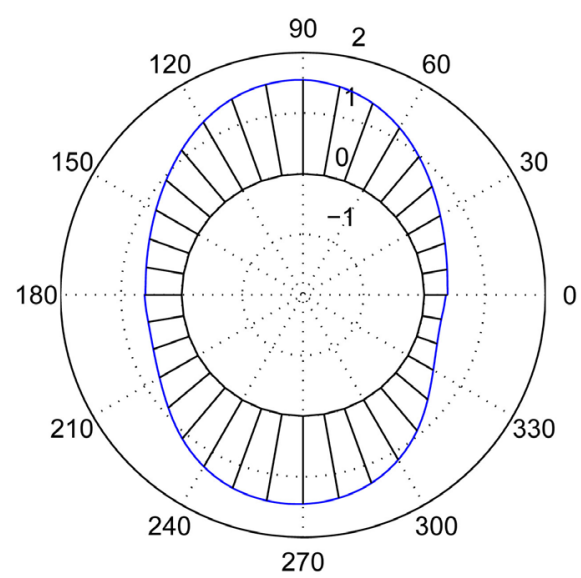

$$
(k a=0.5, a / h=0.5, L / a=2.5)
$$

Figure 6. Dynamic moment factor 6. 


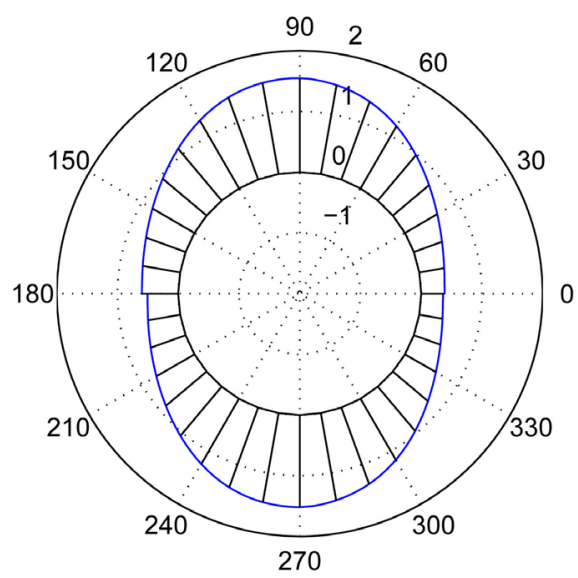

$$
(k a=0.5, a / h=0.5, L / a=4.0)
$$

Figure 7. Dynamic moment factor 7.

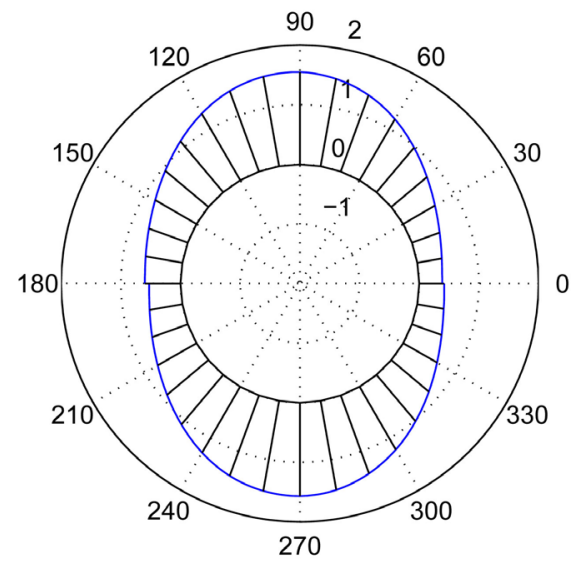

$$
(k a=0.5, a / h=0.5, L / a=6.0)
$$

Figure 8. Dynamic moment factor 8.

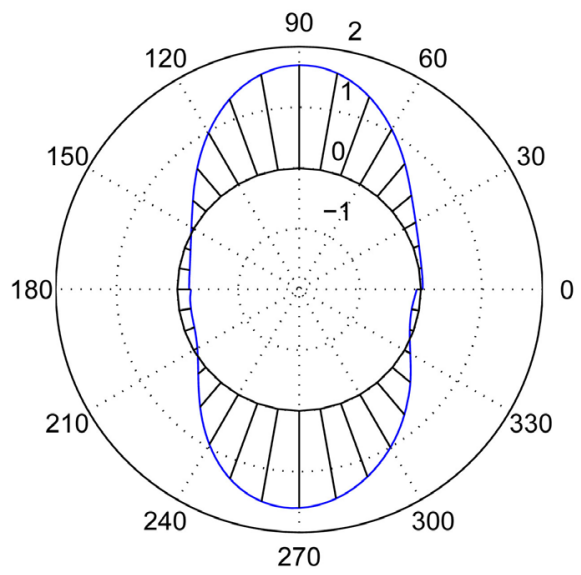

$$
(k a=1.0, a / h=1.0, L / a=2.1)
$$

Figure 9. Dynamic moment factor 9. 


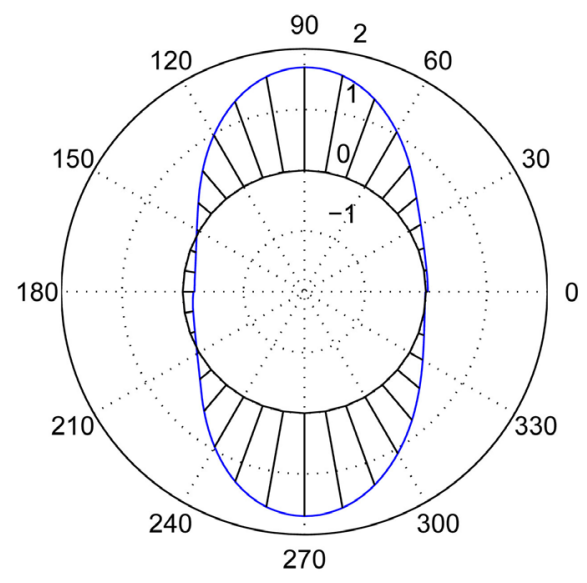

$$
(k a=1.0, a / h=1.0, L / a=2.5)
$$

Figure 10. Dynamic moment factor 10.

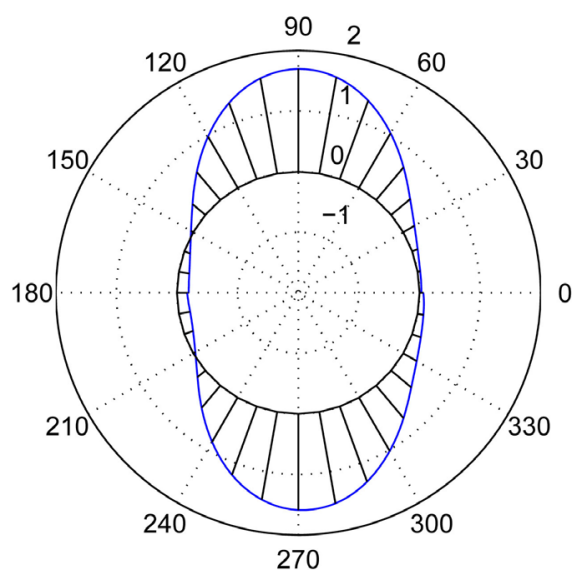

$$
(k a=1.0, a / h=1.0, L / a=4.0)
$$

Figure 11. Dynamic moment factor 11.

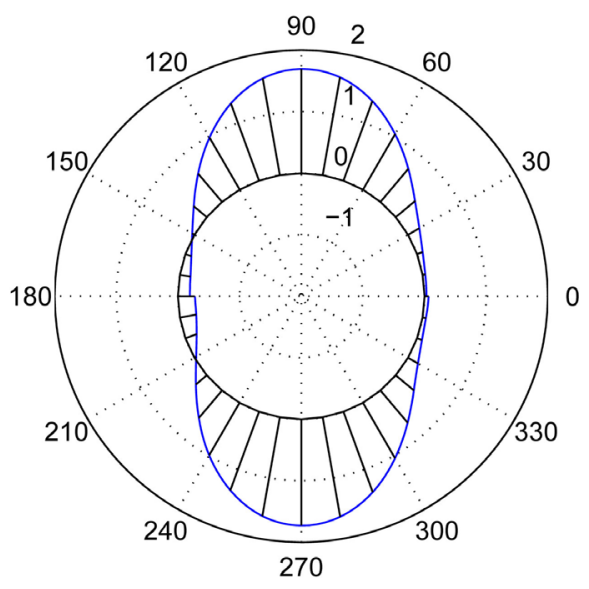

$$
(k a=1.0, a / h=1.0, L / a=6.0)
$$

Figure 12. Dynamic moment factor 12. 


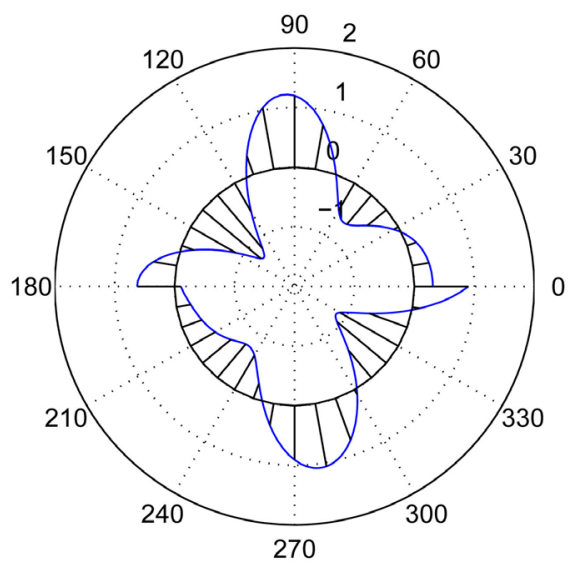

$(k a=3.0, a / h=2.0, L / a=2.1)$

Figure 13. Dynamic moment factor 13.

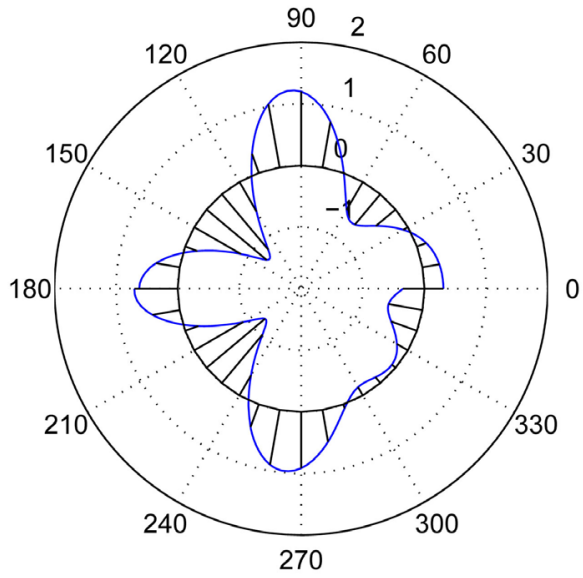

$$
(k a=3.0, a / h=2.0, L / a=2.5)
$$

Figure 14. Dynamic moment factor 14.

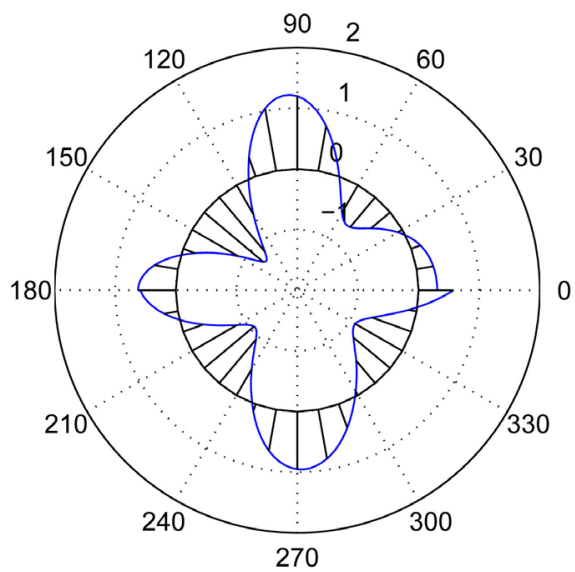

$$
(k a=3.0, a / h=2.0, L / a=4.0)
$$

Figure 15. Dynamic moment factor 15. 


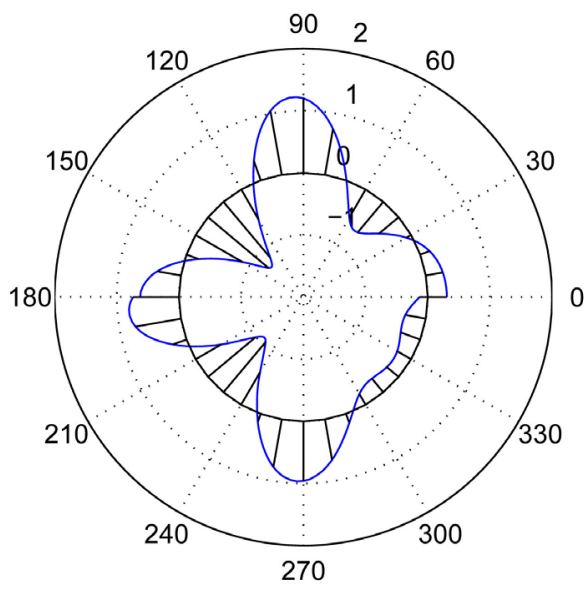

$$
(k a=3.0, a / h=2.0, L / a=6.0)
$$

Figure 16. Dynamic moment factor 16.

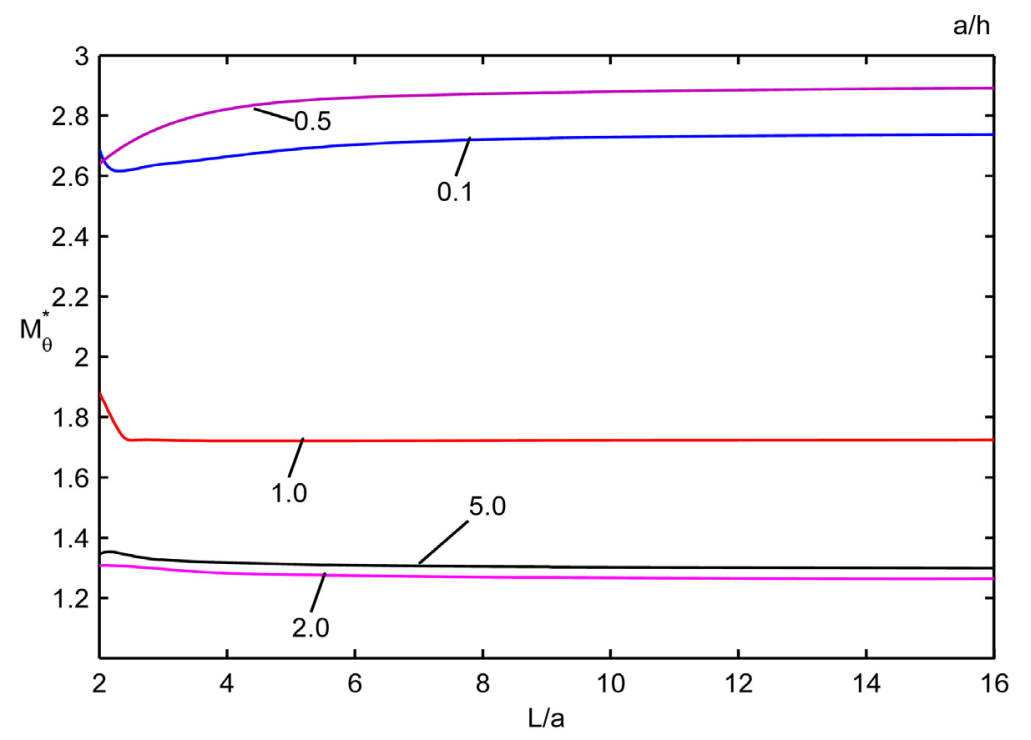

Figure 17. Dynamic moment factors vs dimensionless wave number $(k a=0.1, \theta=\pi / 2)$.

\section{Conclusions}

Based on the precise equation [11] of bending vibration flat, using Liu's complex variable method, the flat hole elastic wave scattering and dynamic stress concentration were studied. Through analysis of the calculation results can be seen:

1) In the case of low incident frequency, dynamic moment concentration coefficient with two holes pitch change is gentle. When the frequency of the incident wave is high, the dynamic moment concentration factor changes with the distance between the two holes, and even a negative bending moment appears.

2) Compared with the literature [10] of the single-hole, because of the interaction between the holes, double holes between the dynamic moments concentrations factors will become more complex. Sometimes actions relieve stress, but sometimes intensify. Therefore, in the process of design, you can not apply the 
full static load strength design standards or specifications should do a comprehensive analysis of dynamic stress.

According to precision plate bending vibration equations [11] that are used in the absence of any works obtained under the hypothetical situation, the kinetic equation is more accurate. Based on the vibration equation, if it needs to be solved by conformal mapping transformation, the analysis problem of two holes of arbitrary shape with dynamic stress concentration can be solved, thereby providing a standardized method. This research method and numerical results are expected to be applied in the thick-walled structural engineering's dynamics analysis and strength design.

\section{Conflicts of Interest}

The authors declare no conflicts of interest regarding the publication of this paper.

\section{References}

[1] Savin, E.H. (1965) Stress Concentrations at the Edge of a Hole. Science Press, Beijing. (In Chinese)

[2] Reissner, E. (1945) The Effect of Transverse Shear Deformation on the Bending of Elastic Plates. ASME Journal of Applied Mechanics, 12, 69-77.

[3] Mindlin, R.D. (1951) Influence of Rotatory Inertia and Shear on Flexural Motions of Isotropic Elastic Plates. Journal of Applied Mechanics, 18, 31-36.

[4] Pao, Y.H. and Mao, C.C., Eds. (1993) Diffraction of Elastic Waves and Dynamic Stress Concentrations. Liu, D.K. and Su, X.Y., Trans. Science Press, Beijing. (In Chinese))

[5] Pao, Y.H. and Mao, C.C. (1964) Diffractions of Flexural Waves by a Cavity in an Elastic Plate. AIAA Journal, 2, 2004-2010. https://doi.org/10.2514/3.2716

[6] Liu, D.K. and Hu, C. (1996) Scattering of Flexural Waves and Dynamic Stress Concentrations in Mindlin Thick Plates with a Cutout. Acta Mechanica Sinica, 12, 169-185. https://doi.org/10.1007/BF02486795

[7] Muskhelishvili, N.I., Ed. (1958) Some Basic Problems in Mathematical Elasticity. Zhao, H.Y., Trans. Science Press, Beijing. (In Chinese)

[8] Liu, D., Gai, B. and Tao, G. (1982) Applications of the Method of Complex Functions to Dynamic Stress Concentrations. Wave Motion, 4, 293-304. https://doi.org/10.1016/0165-2125(82)90025-7

[9] Abramowitz, M. and Stegun, I.A. (1964) Handbook of Mathematical Functions with Formulas, Graphs, and Mathematical Tables. Dover Publications, New York.

[10] Jain, D.L. and Taid, R.P. (1979) Scattering of Elastic Waves by Circular Cylindrical Flaws and Inclusions. Journal of Applied Physics, 50, 4097-4108. https://doi.org/10.1063/1.326489

[11] Jain, D.L. and Taid, R.P. (1980) Scattering of Elastic Waves by an Elastic Sphere. International Journal of Engineering Science, 18, 829-839. https://doi.org/10.1016/0020-7225(80)90029-4

[12] Simon, M.M. and Radlinski, R.P. (1982) Elastic Wave Scattering from Elliptical Shells. Journal of the Acoustical Society of America, 71, 273. https://doi.org/10.1121/1.387451 